\title{
Erratum: Molecular basis of telomere dysfunction in human genetic diseases
}

Grzegorz Sarek, Paulina Marzec, Pol Margalef \& Simon J Boulton

Nat. Struct. Mol. Biol. 22, 867-874 (2015); published online 4 November 2015; corrected after print 20 April 2017

The schematic of the TERT subunit in Figure 1 incorrectly identified the 'Internal RNA template': it now correctly indicates the telomerase RNA. The RNA template sequence has been corrected from AAUCCCAAU to AAUCCCAAUC, and the DNA sequence of the repeat synthesis product added to the $3^{\prime}$ overhang was changed from GGGTTA to GGTTAG. These errors have been corrected in the HTML and PDF versions of the article.

\section{Corrigendum: Quaternary contact in the initial interaction of CD4 with the HIV-1 envelope trimer}

Qingbo Liu, Priyamvada Acharya, Michael A Dolan, Peng Zhang, Christina Guzzo, Jacky Lu, Alice Kwon, Deepali Gururani, Huiyi Miao, Tatsiana Bylund, Gwo-Yu Chuang, Aliaksandr Druz, Tongqing Zhou, William J Rice, Christoph Wigge, Bridget Carragher, Clinton S Potter, Peter D Kwong \& Paolo Lusso

Nat. Struct. Mol. Biol. 24, 370-378 (2017); published online 20 February 2017; corrected after print 27 April 2017

In the version of this article initially published, funding information for B.C. and C.S.P. was missing NIH grant S10 OD019994-01. In addition, there was an incorrect comma in the introduction (after "glycoprotein" in the sentence "Upon binding to the primary cellular receptor, CD4, the external gp120 Env glycoprotein undergoes major conformational changes...") that has been removed. The errors have been corrected in the HTML and PDF versions of the article. 